DFUPG 1/93

\title{
SU $(N)$ ANTIFERROMAGNETS AND STRONGLY COUPLED QED: EFFECTIVE FIELD THEORY FOR JOSEPHSON JUNCTIONS ARRAYS
}

\author{
M. C. Diamantini ${ }^{1}$ and P. Sodano円 \\ Dipartimento di Fisica and Sezione I.N.F.N., Universitá di Perugia \\ Via A. Pascoli, 06100 Perugia, Italy \\ E. Langmann" and G. W. Semenoff \\ Department of Physics, University of British Columbia \\ Vancouver, B.C., Canada V6T 1 Z1 \\ To appear in "Common Trends in Condensed Matter and High Energy \\ Physics" \\ Chia Laguna, Italy, September 1992
}

\begin{abstract}
We review our analysis of the strong coupling limit of compact QED on a lattice with staggered Fermions. We show that, for infinite coupling, compact QED is exactly mapped in a quantum antiferromagnet. We discuss some aspects of this correspondence relevant for effective field theories of Josephson junctions arrays.

\footnotetext{
${ }^{1}$ This work is supported in part by a grant from the M.U.R.S.T.. M.C.D. and P.S. acknowledge the hospitality of the Physics Department at the University of British Columbia where some of this work was completed.

${ }^{2}$ Work supported in part by the "Fonds zur Förderung der wissenschaftlichen Forschung" of Austria under contract Nr. J0789-PHY.

${ }^{3}$ This work is supported in part by the Natural Sciences and Engineering Research Council of Canada. G.S. acknowledges the hospitality of the Physics Department of the University of Perugia and I.N.F.N., Sezione di Perugia where part of this work was completed.
} 


\section{Introduction}

The strong coupling limit of gauge theories such as QCD exhibits many of the qualitative features of their spectrum. For example, confinement is manifest and also, in some cases, it is straightforward to show that chiral symmetry is broken. Recently this issue has been re-examined in detail [1], 2] and the possibility for chiral symmetry breaking for a wide variety of gauge theories has been explored.

It has been recognized for some time that the strong coupling limit of lattice gauge theory with dynamical fermions is related to certain quantum spin systems. This is particularly true in the Hamiltonian picture and appeared in some of the earliest analyses of chiral symmetry breaking in the strong coupling limit [3]. In that work, the chiral symmetry breaking phase of strong coupling gauge theory was analyzed by drawing an analogy with quantum antiferromagnets and then using mean field theory and spin-wave analysis to compute the properties of the pion spectrum.

In more recent literature [4], it has been noted that there are several formal similarities between some condensed matter systems with lattice fermions (particularly certain antiferromagnetic spin systems) and lattice gauge theory systems, usually in their strong coupling limit. For example, it is well known that the quantum spin 1/2 Heisenberg antiferromagnet is equivalent to the strong coupling limit of a particular U(1) lattice gauge theory [5]. It can also be written as a kind of $\mathrm{SU}(2)$ lattice gauge theory [6].

An important feature of the relationship of lattice gauge theory with spin systems is the fact that the staggered Fermions which are used to put the Dirac operator on a lattice resemble ordinary lattice fermions used in tight binding models in condensed matter physics when the latter have a half-filled band and are placed in a background $\mathrm{U}(1)$ magnetic field $\pi(\bmod 2 \pi)(1 / 2$ of a flux quantum) through every plaquette of the lattice. This is an old result for $d=2+1$ [7] where it was already recognized in the first work on the Azbel-Wannier-Hofstaeder problem and has since been discussed in the context of the so-called flux phases of the Hubbard and quantum Heisenberg models. It is actually true for all $d \geq 2+1$ and this equivalence was used in

[1], 2] to find mappings between strong coupling lattice gauge theories with a wide variety of fermion contents and various quantum spin systems.

A place in condensed matter where the magnetic flux which is necessary to 
produce a relativistic spectrum for the fermions can be achieved is in the flux phases [4]. In this case the magnetic field comes from a certain condensate.

Alternatively, as an external magnetic field, for ordinary lattice spacings, it is as yet an experimentally inaccessible flux density. Flux densities of this order of magnitude can presently only be achieved in analog experiments where macroscopic arrays of Josephson junctions take the place of atoms at lattice sites. These systems are described in mean field theory by tight binding electrons in a background $\mathrm{U}(1)$ magnetic field [8, 9] with accuracy increasing with $d[10]$. For $d=2$ their ground state is expected to be a flux phase [11, 12]. The low energy limit in this phase resembles strongly coupled QED.

In the following we shall review some of the features of strongly coupled QED which are useful in mapping the strong coupling limit onto spin systems. We shall also emphasize some of those features which we feel are valuable in formulating effective field theories for Josephson junction arrays. Let us begin with a brief summary of the physics of the latter arrays. In the classical limit they are described by the effective Hamiltonian

$$
H=-J \sum_{<i, j>} \cos \left(\theta_{i}-\theta_{j}+a_{i j}\right)
$$

where $\theta_{i}$ are phases of the superconducting order parameter at the $i$ 'th lattice site (which is an island of superconducting material joined by a weak connection to other similar islands), $a_{i j}$ is proportional to the line integral of the electromagnetic vector potential along the connection between sites $i$ and $j$,

$$
a_{i j}=\frac{2 e}{\hbar c} \int_{i}^{j} A \cdot \mathrm{d} l
$$

Also, $J$ is a coupling constant which is generally a function of external magnetic field and temperature and the summation is over nearest neighbors.

The uniformly frustrated system occurs when the external magnetic field is a constant in each plaquette (where we assume that the islands lie on a two dimensional lattice),

$$
\sum_{\square} a_{i j}=2 \pi f
$$

and $f$ is the ratio between the magnetic flux threading the plaquette and the unit flux. The latter system can be mapped onto a classical Coulomb gas 
problem with Hamiltonian [13]

$$
H=-\pi J \sum_{r, r^{\prime}}[m(r)+f] G\left(r-r^{\prime}\right)\left[m\left(r^{\prime}\right)+f\right]
$$

where the charges $m(r)$ reside on the dual lattice, are restricted to integer values $(m \in \mathbb{Z})$ and the total charge is constrained as

$$
\frac{1}{|V|} \sum_{r} m(r)=f
$$

( $|V|$ is the total number of lattice sites). In $D=2$ the charges represent vortices in the phase field of the superconducting order parameter. Even in that case, the ground state of this system is known only for some particular filling fractions $f$ which depend on the lattice. For example, on a square lattice the ground states are known for some rational filling fraction $f=\frac{p}{q}$, $p, q \in \mathbb{Z}\left[10\right.$, 14]. Of particular interest to us is the fully frustrated case, $f=\frac{1}{2}$ on a square lattice. There, the ground state is a staggered configuration with alternating $m+f=\frac{1}{2}$ and $m+f=-\frac{1}{2}$ on neighboring sites.

Also, for this charged plasma in the continuum, it is known that the ground state of a classical Coulomb gas of this kind is a triangular Wigner lattice [10]. Furthermore, when $f=\frac{1}{2}$ there is a $\mathrm{U}(1)$ symmetry and the ground state exhibits a discrete symmetry $\mathbb{Z}_{2}$. At zero temperature the system chooses one of the two phases.

It has also been shown that the equation which determines the configuration of the order parameter is equivalent to the equation which determines the spectrum of electrons in a lattice in a background magnetic field, the so-called Azbel-Wannier-Hofstaeder problem [8, 9].

These classical systems are the limit of a quantum system where the variables conjugate to the phases of the order parameters are the electric charges residing on the islands

$$
\left[\theta_{i}, Q_{j}\right]=2 i e \hbar \delta_{i j}
$$

The effective Hamiltonian is

$$
H=\frac{1}{2} \sum_{i, j}\left(Q_{i}-Q_{x i}\right) C_{i j}^{-1}\left(Q_{j}-Q_{x j}\right)-J \sum_{<i, j>} \cos \left(\phi_{i}-\phi_{j}+a_{i j}\right)
$$


where $Q_{i}$ is the electric charge and $Q_{x i}$ is an offset, or background charge residing on island $i$. The Coulomb interaction of the charges is described by the capacitance matrix $C_{i j}$. The classical Hamiltonian is regained in the limit $C_{i j} \rightarrow \infty$ or $J \rightarrow \infty$.

Another model which is often compared with (5), and in fact which has been argued to describe the critical behavior of non-classical junction arrays [15], is the Bose-Hubbard model with Hamiltonian

$$
H=-\frac{J}{2} \sum_{<i, j>}\left(\Phi_{i}^{\dagger} e^{i a_{i j}} \Phi_{j}+\text { h.c. }\right)+\frac{1}{2} \sum_{i j} V_{i j} n_{i}\left(n_{j}-\delta_{i j}\right)-\mu \sum_{i} n_{i}
$$

where $\Phi_{i}$ and $\Phi_{i}^{\dagger}$ are the annihilation and creation operator for a boson at site $i$, respectively and $n_{i}=\Phi_{i}^{\dagger} \Phi_{i}$ is the boson number at site $i$. The total number of bosons is controlled by the chemical potential $\mu \leq 0$. The on-site part of the self-interaction vanishes if the site is occupied by a single boson. The energy for more than one boson on a site should be large, to give the bosons a hard core. With hard core bosons, this model is equivalent to the frustrated quantum XY model [12].

There is also a mapping of the frustrated quantum XY model onto a lattice Chern-Simons theory coupled to Fermions [12]. For the uniformly frustrated case, the mean field theory of the latter system is equivalent to lattice fermions in an external field, related to the frustration parameter. The fermion spectrum is that of the Hofstaeder problem and is similar to the fermion system encountered in the flux phase of the Hubbard model.

It is an interesting question to what extent these models share other features of a lattice field theory such as quantum electrodynamics. If the fermion spectrum is relativistic and, given that the models have a $U(1)$ gauge invariance, one might expect dynamical effects to generate the relevant operators of electrodynamics. Furthermore, the infinite coupling limit of lattice QED reduces to the problem of solving for the ground state of a classical coulomb gas with a logarithmic potential, identical to the classical limit of the junction array problem. The ground state of this coulomb gas system has been examined in both contexts in previous literature.

It is interesting to speculate that, when we move away from the classical limit in the case of junction arrays, and when we move away from the infinite coupling limit in the case of compact QED, we still have systems with many common features. For example, both systems have a first order phase 
transition. In the case of the juction array it is the transition which destroys superconductivity. In the case of compact electrodynamics it is where chiral symmetryt, which is broken in the strong coupling limit, is restored. It is accompanied by a confinement-deconfinement transition for electric charges. In the coulomb gas representation of the classical junction array the phase transition corresponds to melting of the Wigner lattice to form a two dimensional plasma. In compact electrodynamics the phase transition restores full translation invariance of the lattice theory (corresponding to chiral invariance in the continuum theory) and the electrons are deconfined. In the latter case the strong coupling, confining ground state has the structure of a Wigner lattice of electrons.

In the following we shall review some aspects of strongly coupled lattice QED relevant to an effective field theory of Josephson junction arrays. We shall show how the analogy between Dirac fermions and tight-binding condensed matter fermions is used to find an exact mapping of the strong coupling limit of compact lattice QED with dynamical staggered fermions onto certain quantum $\mathrm{SU}(N)$ antiferromagnets. The precise structure of the resulting antiferromagnet depends on the number of fermion flavors. We shall also review the argument [1, 2] by which QED with staggered Fermions in any number of spatial dimensions, $D>1$, resembles a condensed matter system of lattice electrons in an external magnetic field and with a half-filled band .

Of course, the understanding of the phase structure, particularly the confining and chiral symmetry breaking phases, and nature of phase transitions of QED is of great fundamental interest to particle physicists. These issues have been studied in the continuum quantum electrodynamics where the possibility of a phase transition in the strong coupling regime is suggested by approximate analytical results using the quenched ladder approximation. The idea for the mechanism, which has long been advocated by Miranski and collaborators [16, 17], is that when the electric charge reaches a certain critical value the vacuum of QED becomes unstable, leading to a sort of collapse phenomenon, analogous to the sparking of the vacuum in the presence of a supercritical nucleus. In the picture which we propose, the analog of the collapse phenomenon is the formation of either charge or isospin density waves

\footnotetext{
${ }^{4}$ We shall see in following sections that, for staggered fermions, chiral symmetry corresponds to invariance under translations by one lattice site
} 
and the resulting reduction of the lattice translation symmetry from translations by one site to translations by two sites. It occurs when the exchange interaction of the electrons, which is attractive, dominates the tendency of the direct Coulomb interaction and the kinetic energy to delocalize charge, giving an instability to the formation of commensurate charge density waves. This forms a gap in the Fermion spectrum and a particular mass operator obtains a vacuum expectation value. This gives an intuitive picture of how strong attractive interactions in a field theory can form a coherent structure. Here, the commensurate density waves in the condensed matter system correspond to a modulation of the vacuum charge or isospin density at the ultraviolet cutoff wavelength in the field theory.

In the continuum, we are generally interested in massless quantum electrodynamics with the action

$$
S=\int d^{D+1} x\left(-\frac{\Lambda^{3-D}}{4 e^{2}} F_{\mu \nu} F^{\mu \nu}+\sum_{a=1}^{N_{F}} \bar{\psi}^{a} \gamma^{\mu}\left(i \partial_{\mu}+A_{\mu}\right) \psi^{a}\right)
$$

where $\Lambda$ is the ultraviolet cutoff, $e$ is the dimensionless electric charge and there are $N_{F}$ flavors of $2^{[(D+1) / 2]}-$ dimensional Dirac spinors. (Here, $[(D+1) / 2]$ is the largest integer less than or equal to $(D+1) / 2$.) In even dimensions (when $\mathrm{D}+1$ is even), the flavor symmetry is $\mathrm{SU}_{R}\left(N_{F}\right) \times \mathrm{SU}_{L}\left(N_{F}\right)$. In odd dimensions there is no chirality and the flavor symmetry is $\operatorname{SU}\left(N_{F}\right)$. What is usually referred to as chiral symmetry there is a combination of parity and a $\mathbb{Z}_{2}$ subgroup of the flavor group. We shall use a lattice regularization of (7) and study the limit as $e^{2} \rightarrow \infty$. We shall use $N_{L}$ flavors of staggered fermions. In the naive continuum limit, this gives $N_{F}=N_{L}$ 2-component fermions in $D=1, N_{F}=2 N_{L} \quad 2$-component fermions in $D=2$ and $N_{F}=2 N_{L}$ component fermions in $D=3$. Though the lattice theory reduces to (7) in the naive continuum limit, the lattice regularization breaks part of the flavor symmetry. All of the operators which are not symmetric are irrelevant and vanish as the lattice spacing is taken to zero. In $D=3$, the $\operatorname{SU}_{R}\left(N_{F}\right) \times \mathrm{SU}_{L}\left(N_{F}\right)$ symmetry is reduced to $\mathrm{SU}\left(N_{F} / 2\right.$ ) (in $D=3, N_{L}=N_{F} / 2$ ) and translation by one site in the 3 lattice directions. These discrete transformations correspond to discrete chiral transformations in the continuum theory. In $D=2$, the lattice has $\mathrm{SU}\left(N_{F} / 2\right.$ ) (in $\left.D=2, N_{L}=N_{F} / 2\right)$ symmetry and two discrete ("chiral") symmetries. In $D=1$, the $\mathrm{SU}_{L}\left(N_{F}\right) \times \mathrm{SU}_{R}\left(N_{F}\right)$ symmetry is reduced to $\mathrm{SU}\left(N_{F}\right)$ (in $D=1, N_{L}=N_{F}$ ) and a discrete chiral transformation. 
In each case, the discrete chiral symmetry is enough to forbid Fermion mass and it is the spontaneous breaking of this symmetry which we shall examine and which we shall call "chiral symmetry breaking". We shall also consider the possibility of spontaneous breaking of the $\mathrm{SU}\left(N_{F}\right)$ flavor symmetry. In the continuum the $\mathrm{SU}\left(N_{F}\right)$ corresponds to a vector-like symmetry subgroup of the full flavor group. Since the lattice and continuum theories have different symmetries, the spectrum of Goldstone bosons, etc. would be different in the two cases. We shall not address the source of these differences but will define QED by its lattice regularization and discuss the realization of the symmetries of that theory only.

In the strong coupling limit, lattice QED with $N_{\mathrm{L}}$ lattice flavors of staggered Fermions is exactly equivalent to an $\mathrm{SU}\left(N_{\mathrm{L}}\right)$ quantum antiferromagnet where the spins are in a particular fundemental representation of the $\mathrm{SU}\left(N_{\mathrm{L}}\right)$ Lie algebra. Furthermore, mass operators of QED correspond to staggered charge and isospin density operators in the antiferromagnet. Thus, the formation of charge-density waves corresponds to chiral symmetry breaking and the dynamical generation of an iso-scalar Fermion mass whereas Néel order of the antiferromagnet corresponds to dynamical generation of Fermion mass with an iso-vector condensate. As a result of this correspondence we are able to use some of the properties of the quantum antiferromagnets to deduce features of strongly coupled QED.

In the infinite coupling limit, the properties of the electronic ground state of compact and non-compact QED are identical. The fact that compact QED confines and non-compact QED (at least in high enough dimensions) does not confine affects only the properties of the gauge field wavefunctions and the elementary excitations. In the following we shall focus our attention only on compact QED.

An interesting result of our approach [2] is that the case of even $N_{\mathrm{L}}$ and odd $N_{\mathrm{L}}$ are very different:

When $N_{\mathrm{L}}$ is odd, the vacuum energy in the strong coupling limit is proprotional to $e^{2}$, the square of the electromagnetic coupling constant. We also find that chiral symmetry is broken in the strong coupling limit for all odd $N_{\mathrm{L}} \geq 1$ and for all spacetime dimensions $D+1 \geq 2$. The mass operator which obtains a nonzero expectation value is a Lorentz and flavor Lie algebra scalar. There are also mass operators which are Lorentz scalars and which transform nontrivially under the flavor group which could get an expectation 
value and break the flavor symmetry spontaneously if $N_{\mathrm{L}}$ is small enough. When $N_{\mathrm{L}}$ increases to some critical value there is a phase transition to a disordered phase [18].

In contrast, when $N_{\mathrm{L}}$ is even we find that the vacuum energy in the strong coupling limit is of order 1 . We find that chiral symmetry is broken when the spacetime dimension $D+1 \geq 3$ and when $N_{\mathrm{L}}$ is small enough. The mass operator which gets an expectation value is a Lorentz scalar and transforms nontrivially under the flavor $\mathrm{SU}\left(N_{\mathrm{L}}\right)$. Thus, flavor symmetry is spontaneously broken. As in the case of odd $N_{\mathrm{L}}$, there is an upper critical $N_{\mathrm{L}}$ where there is a transition to a disordered phase [18].

For $N_{\mathrm{L}}$ odd finding the gauge invariant electronic ground state of strongly coupled lattice QED is equivalent [2] to finding the exact ground state of the generalized classical Coulomb gas model in $D$-dimensions with Hamiltonian

$$
H_{\text {coul }}=\frac{e^{2}}{2} \sum_{<x, y>} \rho(x) g(x-y) \rho(y)
$$

where the variable $\rho(x)$ lives at the sites of a cubic lattice with spacing one (with coordinates $\left(x_{1}, \ldots, x_{D}\right)$ and $x_{i}$ are integers) and takes on values

$$
-\frac{N_{\mathrm{L}}}{2},-\frac{N_{\mathrm{L}}}{2}+1, \ldots, \frac{N_{\mathrm{L}}}{2}+1, \frac{N_{\mathrm{L}}}{2} \quad N_{\mathrm{L}} \text { an odd integer }
$$

and the interaction is long-ranged

$$
g(x-y) \sim|x-y|^{2-D} \quad, \quad \text { as }|x-y| \rightarrow \infty
$$

In any space dimension, $D \geq 1$ we prove that there are two degenerate ground states which have the Wigner lattice configurations

$$
\rho(x)= \pm \frac{1}{2}(-1)^{\sum_{i=1}^{D} x_{i}}
$$

When $N_{\mathrm{L}}=1$ this is a long-ranged Ising model which is known to have antiferromagnetic order, even in one dimension. It is also in agreement with a known result about the Ising model in two dimensions [10].

The Coulomb gas representation of the classical Josephson junction array is related to this system when $f=1 / 2$, i.e. in the fully frustrated case. There, the charge on a site is only limited by dynamics, whereas in electrodynamics it 
is restricted by the Pauli principle to be between $-N_{L} / 2$ and $N_{L} / 2$. However, in the case of the classical Coulomb gas, the Coulomb energy of more than one charge residing on a lattice site is infinite, so effectively, whenever $f<1$ the charges at lattice sites should also be either 1,0 or -1 . Therefore the problem of determining the charge (vortex) distribution in a fully frustrated junction array is equivalent to finding the ground state of infinite coupling QED with $N_{L}=1$. It will turn out that the solution of this problem is similar for all odd $N_{L}$.

The difference between even and odd $N_{\mathrm{L}}$ can be seen to arise from a certain frustration encountered when poses the conditions on lattice QED which should lead to charge conjugation invariance, translation invariance and Lorentz invariance of the continuum limit. This frustration is absent when $N_{\mathrm{L}}$ is even. This is not an anomaly in the conventional sense of the axial anomaly or a discrete anomaly encountered in the quantization of gauge theories, as no symmetries are incompatible, but it is nevertheless an interesting analog of the anomaly phenomenon.

Note that in $2+1$ dimensions the difference between even and odd $N_{\mathrm{L}}$ is the difference between an even and odd number of 4-component Fermions. Our result that for an odd number of 4-component Fermions the chiral symmetry is always broken for large coupling seems to contradict the continuum analysis in 19, 20]. We do not fully understand the reason for this, but speculate that it is related to the lattice regularization. Their model is very similar to ours in that they effectively work in the strong coupling limit when they replace the ultraviolet regularization which comes from having a Maxwell term in the QED action by a large momentum cutoff. In our case we have a lattice cut-off and the result should be very similar. Note that we agree with them when $N_{\mathrm{L}}$ is even and there is an even number of 4-component Fermions. In that case, we are also in qualitative agreement with recent numerical work [21] which, since it uses Euclidean staggered Fermions and there is a further Fermion doubling due to discretization of time, can only consider the case where there is an even number of 4-component Fermions. The anomalous behavior that we find with odd numbers of 4-component Fermions exposes a difficulty with treating the flavor number $N$ as a continuous parameter.

A hint as to why 2+1-dimensional Fermions should come in four-component

\footnotetext{
${ }^{5}$ Note that this frustration is different from that caused by the external magnetic field in the junction array
} 
units appears when we formulate compact QED in the continuum using the SO(3) Georgi-Glashow model with spontaneous breaking of the global symmetry, $\mathrm{SO}(3) \rightarrow \mathrm{U}(1)$ and the limit of large Higgs mass [22]. We begin with the model

$$
\mathcal{L}=\frac{1}{4 e^{2}} \sum_{a=1}^{3}\left(F_{\mu \nu}^{a}\right)^{2}+\frac{1}{2} \sum_{a=1}^{3}(D \phi)^{a} \cdot(D \phi)^{a}+\frac{\lambda}{4}\left(\left(\sum_{a=1}^{3} \phi^{a} \phi^{a}\right)-v^{2}\right)^{2}
$$

The spectrum contains a massless photon which in the $\lambda \rightarrow \infty$ and $v^{2} \rightarrow \infty$ is the only light excitation. Since the U(1) gauge group is a subgroup of $\mathrm{SO}(3)$, it is compact. We wish to make $2+1$-dimensional electrodynamics by coupling this model to Fermions. It is known that, if the resulting theory is to preserve parity and gauge invariance simultaneously, we must use an even number of two-component Fermions which, in the minimal case are also $\mathrm{SU}(2)$ doublets [23]

$$
\mathcal{L}_{F}=\bar{\psi}\left(i \gamma \cdot \nabla+\gamma \cdot A+\gamma^{5}(m+g \phi \cdot \sigma)\right) \psi
$$

Here, the Fermions are four-component and have a parity invariant mass and Higgs coupling. Each SU(2) doublet contains two electrons (which can be defined so that both components of the doublet have the same sign of electric charge), thus the basic Fermion for compact QED has eight components. The maximal chiral symmetry is obtained in the massless case with vanishing Higgs coupling. It is possible, by suitable choice of mass and Higgs coupling i.e. $m= \pm g\langle\phi\rangle$, (and also reduction of the chiral symmetry) to make four of the Fermions heavy, leaving four massless components. This is consistent with parity and gauge invariance. It is also interesting to note that staggered Fermions on a Euclidean lattice, where time is also discretized, produce eight component continuum Fermions.

In Section 2 we review the essential features of the staggered fermion formalism. In Section 3 we elucidate how spin algebras may be represented by Fermion bilinears. In Section 4 we formulate lattice QED and analyze its discrete symmetries. Section 5 is devoted to the analysis of the strong coupling limit of compact QED. There we shall review the properties of the electronic ground state for both $N_{\mathrm{L}}$ even and odd. Section 6 is devoted to concluding remarks. 


\section{Staggered Fermions}

Here we make explicit the fact that latticized (staggered) relativistic fermions are equivalent to the Hofstaeder problem with flux density $1 / 2$ flux quanta per plaquette.

In the following, we use units in which the lattice spacing, the speed of light and Planck's constant are all equal to one. Space is a $D$-dimensionsal cubic lattice with lattice points $x=\left(x_{1}, x_{2}, \ldots, x_{D}\right)\left(x_{i}\right.$ integers $)$, oriented links $[x, i]$ labeled by their site of origin and their direction, $i= \pm 1, \ldots, \pm D$, and oriented plaquettes $[x, i, j]$ with corners $x, x+\hat{i}, x+\hat{i}+\hat{j}, x+\hat{j}$ and orientation $\hat{i} \times \hat{j}$ where $\hat{i}$ is the unit vector in $i$-direction.

In relativistic field theory, the purpose of the staggered Fermion method is to minimize Fermion doubling which always occurs when one tries to put Fermions on the lattice. Generally, staggered Fermions are obtained by the spin-diagonalization method. To implement this method, we begin with the naively latticized Dirac Hamiltonian for free relativistic fermions,

$$
H_{F}=\frac{1}{2} \sum_{[x, j]}\left(\psi^{\dagger}(x) i \alpha^{j} \nabla_{j} \psi(x)-\left(\nabla_{j} \psi^{\dagger}\right)(x) i \alpha^{j} \psi(x)\right)
$$

where the lattice derivative operator is defined as difference operator

$$
\nabla_{i} f(x)=f(x+\hat{i})-f(x)
$$

and $\alpha^{j}$ are the $2^{[(D+1) / 2]}$-dimensional Dirac $\alpha$-matrices. (Here $[(D+1) / 2]$ is the integer part of $(D+1) / 2$.) The latter are Hermitean, $\alpha^{j \dagger}=\alpha^{j}$, and obey the Clifford algebra

$$
\left\{\alpha^{i}, \alpha^{j}\right\}=2 \delta^{i j}
$$

They are therefore unitary matrices, $\alpha^{i \dagger} \alpha^{i}=1$. Using the properties of the difference operator, (14) can be presented in the form

$$
H_{F}=-\frac{i}{2} \sum_{[x, j]}\left(\psi^{\dagger}(x+\hat{j}) \alpha^{j} \psi(x)-\psi^{\dagger}(x) \alpha^{j} \psi(x+\hat{j})\right)
$$

Since the Dirac matrices are unitary, the naive lattice Fermion Hamiltonian in (16) resembles a condensed matter Fermion hopping problem with a background $\mathrm{U}\left(2^{[(D+1) / 2]}\right)$ gauge field given by the $\alpha$-matrices. In any plaquette of 
the lattice, $[x, i, j]$, this background field has curvature

$$
\alpha^{i} \alpha^{j} \alpha^{i \dagger} \alpha^{j \dagger}=-1
$$

The curvature resides in the $\mathrm{U}(1)$ subgroup of $\mathrm{U}\left(2^{[(D+1) / 2]}\right)$ and has exactly half of a $U(1)$ flux quantum per plaquette. This is true in any dimensions. We observe that either $1 / 2$ or zero flux quanta are the only ones allowed by translation invariance and parity and time reversal symmetries of the Hamiltonian.

Since the curvature of the $\alpha$-matrices is $\mathrm{U}(1)$-valued, we should be able to do a gauge transformation which presents the matrices themselves as $\mathrm{U}(1)$ valued gauge fields (i.e. diagonal). A specific example of such a gauge transformation due to Kluberg-Stern et.al. [24] is

$$
\psi(x) \rightarrow\left(\alpha^{1}\right)^{x_{1}}\left(\alpha^{2}\right)^{x_{2}} \ldots\left(\alpha^{D}\right)^{x_{D}} \psi(x)
$$

Then

$$
\psi^{\dagger}(x+\hat{j}) \alpha^{j} \psi(x) \rightarrow(-1)^{\sum_{k=1}^{j-1} x_{k}} \psi^{\dagger}(x+\hat{j}) \psi(x)
$$

The resulting Hamiltonian is

$$
H_{F}=-\frac{i}{2} \sum_{[x, j]}(-1)^{\sum_{k=1}^{j-1} x_{k}}\left(\psi^{\dagger}(x+\hat{j}) \psi(x)-\psi^{\dagger}(x) \psi(x+\hat{j})\right)
$$

This describes $2^{[(D+1) / 2]}$ identical copies of Fermions with the same Hamiltonian which must all give Fermions with the same spectrum as the original Hamiltonian in (14). Staggered Fermions are obtained by choosing one of these copies. This reduces the Fermion doubling by a factor of the dimension of the Dirac matrices, $2^{[(D+1) / 2]}$.

In the staggered Fermion method, we treat the components of the original lattice Dirac Hamiltonian as flavors, rather than components of the relativistic spinor necessary for Lorentz invariance. The spinor components now reside on adjacent lattice sites. In this method, the continuous chiral symmetry of the massless Hamiltonian, under the transformation $\psi \rightarrow e^{i \gamma^{5} \theta} \psi$, is lost. There is a discrete chiral symmetry, corresponding to translations by one lattice site in any direction. Explicitly,

$$
\psi(x) \rightarrow(-1)^{\sum_{k=j+1}^{D} x_{k}} \psi(x+\hat{j})
$$


is a symmetry of the Hamiltonian (20) and corresponds to a discrete chiral transformation.

Mass operators correspond to staggered charge densities. The operator

$$
\Sigma=\sum_{x}(-1)^{\sum_{k=1}^{D} x_{k}} \psi^{\dagger}(x) \psi(x)
$$

changes sign under the chiral transformations (21) and corresponds to a certain Dirac mass.

With staggered Fermions there is still a certain amount of Fermion doubling. The doubling can be counted by noting that the staggered Fermion Hamiltonian (20) is invariant under translations by two lattice sites. Therefore, a unit cell is a unit hypercube of the lattice, containing $2^{D}$ sites and staggered Fermions correspond to a $2^{D}$ component spinor. The dimension of the Dirac matrices is $d^{[(D+1) / 2]}$. Therefore the number of Dirac spinors we obtain is $2^{D} / 2^{[(D+1) / 2]}$. For lower dimensions the minimum number of continuum flavors can be tabulated as

$\begin{array}{ccc}d & \text { dim. of Dirac matrices } & \text { No. of flavors } \\ 1+1 & 2 & 1 \\ 2+1 & 2 & 2 \\ 3+1 & 4 & 2\end{array}$

Only in 1+1-dimensions do we get a single species of Dirac Fermion.

To see how to take the continuum limit explicitly, consider the case of $d=3+1$. There, we divide the lattice into eight sublattices and label the spinor components as

$$
\begin{aligned}
& \psi(\text { even, even, even }) \equiv \psi_{1} \quad \psi(\text { odd }, \text { even, odd }) \equiv \psi_{7} \\
& \psi(\text { even, odd }, \text { even }) \equiv \psi_{6} \quad \psi(\text { even, even, odd }) \equiv \psi_{5} \\
& \psi(\text { odd }, \text { odd }, \text { even }) \equiv \psi_{4} \quad \psi(\text { odd }, \text { even, odd }) \equiv \psi_{3} \\
& \psi(\text { even, odd }, \text { odd }) \equiv \psi_{2} \quad \psi(\text { odd }, \text { odd }, \text { odd }) \equiv \psi_{8}
\end{aligned}
$$

In terms of these spinors, the Hamiltonian (20) can be written as the matrix operator

$$
H=\int_{\tilde{\Omega}_{B}} d^{3} k \psi^{\dagger}(k) A^{i} \sin k_{i} \psi(k)
$$


where

$$
\tilde{\Omega}_{B}=\left\{k_{i}:-\pi / 2<k_{i} \leq \pi / 2\right\}
$$

is the Brillouin zone of the (even,even,even) sublattice,

$$
A^{i}=\left(\begin{array}{cc}
0 & \alpha^{i} \\
\alpha^{i} & 0
\end{array}\right)
$$

and

$$
\alpha^{1}=\left(\begin{array}{ll}
0 & 1 \\
1 & 0
\end{array}\right) \quad \alpha^{2}=\left(\begin{array}{cc}
\sigma^{1} & 0 \\
0 & -\sigma^{1}
\end{array}\right) \quad \alpha^{3}=\left(\begin{array}{cc}
\sigma^{3} & 0 \\
0 & -\sigma^{3}
\end{array}\right)
$$

are a particular representation of the Dirac matrices.

In this representation the mass operator is

$$
\sum_{x}(-1)^{\sum_{k=1}^{3} x_{k}} \psi^{\dagger}(x) \psi(x)=\int_{\tilde{\Omega}_{B}} \frac{d^{3} k}{(2 \pi)^{3}} \psi^{\dagger}(k) B \psi(k)
$$

where

$$
B=\left(\begin{array}{cc}
1 & 0 \\
0 & -1
\end{array}\right)
$$

The Fermion spectrum is

$$
\omega(k)=\sqrt{\sum_{i=1}^{3} \sin ^{2} k_{i}+m^{2}}
$$

and only the region $k_{i} \sim 0$ is relevant to the continuum limit. We have normalized $\psi(k)$ so that

$$
\left\{\psi(x), \psi^{\dagger}(y)\right\}=\delta(x-y), \quad\left\{\psi(k), \psi^{\dagger}(l)\right\}=\delta(k-l)
$$

If we define

$$
\beta=\left(\begin{array}{cc}
\sigma^{2} & 0 \\
0 & -\sigma^{2}
\end{array}\right)
$$

and the unitary matrix

$$
M=\frac{1}{2}\left(\begin{array}{ll}
1-\beta & 1+\beta \\
1+\beta & 1-\beta
\end{array}\right)
$$


and

$$
\psi=M \psi^{\prime}
$$

with

$$
\psi^{\prime}=\left(\psi_{a}, \psi_{b}\right)
$$

the Hamiltonian is

$$
H_{f}=\int_{\Omega_{B}} d^{3} k\left(\psi_{a}^{\dagger}, \psi_{b}^{\dagger}\right)\left(\begin{array}{cc}
\alpha^{i} \sin k_{i}-\beta m & 0 \\
0 & \alpha^{i} \sin k_{i}+\beta m
\end{array}\right)\left(\begin{array}{l}
\psi_{a} \\
\psi_{b}
\end{array}\right)
$$

In the low momentum limit, $\sin k_{i} \sim k_{i}$, with Fermion density $1 / 2$ per site so that the Fermi level is at the intersection point of the positive and negative energy bands, we obtain 2 continuum Dirac Fermions.

This describes two flavors of 4-component Dirac Fermions and the Dirac masses for each component given by the staggered charge density have opposite signs. Thus the charge density breaks the discrete chiral symmetry. It also breaks a flavor symmetry which, in the absence of mass, mixes the two continuum Fermions. The continuum limit for arbitrary $d$ is discussed in [2]

\section{Fermion Representation of SU $(N)$ Quan- tum Antiferromagnet}

In order to set the formalism which allows us to find the exact mapping between strongly coupled lattice QED and $\mathrm{SU}(N)$ quantum antiferromagnets, we review here the representation of an $\mathrm{SU}(N)$ antiferromagnet in terms of fermion bilinears. The analysis holds for any space dimension D.

The Hamiltonian for an $\mathrm{U}(N)$ quantum antiferromagnet is

$$
H_{\mathrm{AFM}}=\frac{g^{2}}{2} \sum_{<x, y>} J_{a b}(x) J_{b a}(y)
$$

where $J_{a b}(x), a, b=1, \cdots, N_{\mathrm{L}}$, obey current algebra relations associated with the Lie algebra of $\mathrm{U}(N)$,

$$
\left[J_{a b}(x), J_{c d}(y)\right]=\left(J_{a d}(x) \delta_{b c}-J_{c b}(x) \delta_{a d}\right) \delta(x-y)
$$


and where $\langle x, y\rangle$ denotes the link connecting sites $x$ and $y$ on a bipartite lattice. For simplicity, we take the lattice to be cubic. Here, we have used a particular basis for the $\mathrm{SU}(N)$ algebra which can be conveniently represented by Fermion bilinear operators,

$$
J_{a b}(x)=\psi^{a \dagger}(x) \psi^{b}(x)-\delta^{a b} / 2
$$

with Fermion field operators $\psi^{a(\dagger)}$ obeying

$$
\left\{\psi^{a}(x), \psi^{b \dagger}(y)\right\}=\delta_{a b} \delta(x-y)
$$

The representation of the algebra on each site $x$ is fixed by specifying the Fermion number of the states,

$$
\rho(x)=\sum_{a} J_{a a}(x)
$$

For example, the Fermion vacuum state $\mid 0>$ such that

$$
\psi^{a}(x) \mid 0>=0 \quad, \forall a, x
$$

is the singlet state, the states with $m \leq N$ Fermions per site,

$$
\prod_{x} \psi^{a_{1} \dagger}(x) \psi^{a_{2} \dagger}(x) \ldots \psi^{a_{m} \dagger}(x) \mid 0>
$$

corresponds to $\rho(x)=m-N / 2$ for all $x$ and the irreducible representation with the Young Tableau with one column and $m$ boxes.

For each site $x, \rho(x)$ is the generator of the $\mathrm{U}(1)$ subgroup of $\mathrm{U}(N)$. Using a basis $T^{i}=\left(T^{i}\right)^{*}, i=1, \ldots, N^{2}-1$, of the Lie algebra of $\mathrm{SU}(N)$ in the fundamental representation normalized so that $\operatorname{tr}\left(T^{i} T^{j}\right)=T_{a b}^{i} T_{b a}^{j}=\delta^{i j} / 2$, and using

$$
T_{a b}^{i} T_{c d}^{i}=\frac{1}{2} \delta_{a d} \delta_{b c}-\frac{1}{2 N} \delta_{a b} \delta_{c d}
$$

it is convenient to introduce

$$
J^{i}(x)=\psi^{a \dagger}(x) T_{a b}^{i} \psi^{a}(x)
$$

obeying current algebra of the Lie algebra of $\mathrm{SU}(N)$, and to write the Hamiltonian (40) as

$$
H_{\mathrm{AFM}}=\frac{g^{2}}{N} \sum_{<x, y>} \rho(x) \rho(y)+H_{\mathrm{SU}(N)}
$$


with

$$
H_{\mathrm{SU}(N)}=g^{2} \sum_{<x, y>} J^{i}(x) J^{i}(y)
$$

is the Hamiltonian of an $\mathrm{SU}(N)$ antiferromagnet. From this it is obvious that by fixing the $\rho(x), H_{\mathrm{AFM}}$ is reduced to an $\mathrm{SU}(N)$ antiferromagnet.

For example, the familiar $j=1 / 2 \mathrm{SU}(2)$ Heisenberg antiferromagnet is obtained from (40), $N=2$, by using the identity

$$
\frac{\vec{\sigma}_{a b}}{2} \cdot \frac{\vec{\sigma}_{c d}}{2}=\frac{1}{2} \delta_{a d} \delta_{b c}-\frac{1}{4} \delta_{a b} \delta_{c d}
$$

corresponding to (45) for $N=2$.

Generally, when $N$ is even we will consider the representations where $m=N / 2$, so that the Fermion occupation of each site is $N / 2$ and $\rho(x)=0$. When $N$ is odd we divide the lattice into two sublattices such that the nearest neighbors of all sites of one sublattice are in the other sublattice (when this is possible the lattice is said to be bipartite). When $N$ is odd, the representation of $\mathrm{SU}(N)$ has $(N+1) / 2$ Fermions, i.e. $\rho(x)=1 / 2$, on the sites of one sublattice and $(N-1) / 2$ Fermions, i.e. $\rho(x)=-1 / 2$ on the sites of the other sublattice.

\section{QED on a Lattice}

In this Section we shall review the Hamiltonian formalism of Abelian gauge fields on a lattice. For the most part, this formalism can be found in some of the classic reviews of lattice gauge theory, 25] for example. A novel feature of our approach [2] is a careful treatment of the normal ordering of the charge operator and a discussion of the ensuing discrete symmetries. This normal ordering turns out to be important if the continuum limit has to have the correct behavior under $C, P$ and $T$ transformations. It will also be important in our later solution of the strong coupling limit.

\subsection{Hamiltonian and Gauge Constraint}

We shall discretize space as a cubic lattice and, in order to use the Hamilto-

nian formalism; time is left continuous. Lattice gauge fields are introduced 
through the link operators

$$
U_{i}(x) \equiv e^{i A_{i}(x)}
$$

which correspond to the links $[x, i]$ of the lattice. Electric fields propagate on links of the lattice and the electric field operator $E_{i}(x)$ associated with the link $[x, i]$ is the canonical conjugate of the gauge field

$$
\left[A_{i}(x), E_{j}(y)\right]=i \delta_{i j} \delta(x-y)
$$

The gauge field and electric field operators obey the relations

$$
A_{-i}(x)=-A_{i}(x-\hat{i}) \quad, \quad E_{-i}(x)=-E_{i}(x-\hat{i})
$$

The Hamiltonian of compact QED is

$$
\begin{aligned}
H_{\mathrm{NC}}=\sum_{[x, i]} \frac{e^{2}}{2} E_{i}^{2}(x)+ & \sum_{[x, i, j]} \frac{1}{2 e^{2}} \sin ^{2}(B[x, i, j] / 2) \\
& +\sum_{[x, i]}\left(t_{[x, i]} \psi^{a \dagger}(x+\hat{i}) e^{i A_{i}(x)} \psi^{a}(x)+\text { h.c. }\right)
\end{aligned}
$$

where the second term contains a sum over plaquettes and the magnetic field is defined as the curvature of the gauge field at the plaquette $[x, i, j]$,

$$
\begin{aligned}
B[x, i, j]=A_{i}(x)+ & A_{j}(x+\hat{i})+A_{-i}(x+\hat{i}+\hat{j})+A_{-j}(x+\hat{j}) \\
& =A_{i}(x)-A_{j}(x)+A_{j}(x+\hat{i})-A_{i}(x+\hat{j})
\end{aligned}
$$

As discussed in Section 2, the hopping parameters $t_{[x, i]}$ contains phases which produce a background magnetic flux $\pi$ per plaquette. In the weak coupling continuum limit, the magnitude of $t_{[x, i]}$ is one, $|t|^{2} \equiv\left|t_{[x, i]}\right|^{2}=1$ in order that the speed of the free photon and free electron fields are equal, i.e. so that the low frequency dispersion relations for both the photon and electron have the same speed of light. However, in order to obtain a relativistic continuum limit in general it is necessary to make $|t|$ a function of $e^{2}$. We shall find that in the limit where $e^{2}$ is large, the speed of light is proportional to $|t| / e$ and it is necessary that $|t| \sim e$ as $e^{2} \rightarrow \infty$.

Equation (53) reduces to the standard Hamiltonian of QED in the naive weak coupling continuum limit. In the strong coupling limit compact QED 
is confining [22] and the phase transition associated with chiral symmetry breaking is generally of first order [26]. In (53) we have introduced $N_{\mathrm{L}}$ flavors of lattice Fermions labelled by the index $a=1, \ldots, N_{\mathrm{L}}$.

The Hamiltonian is supplemented with the constraint of gauge invariance. The gauge transformations of the dynamical variables,

$$
\begin{array}{ll}
\Lambda: & A_{i}(x) \rightarrow A_{i}(x)+\nabla_{i} \Lambda(x) \\
\Lambda: & E_{i}(x) \rightarrow E_{i}(x) \\
\Lambda: & \psi^{a}(x) \rightarrow e^{i \Lambda(x)} \psi^{a}(x) \\
\Lambda: & \psi^{a \dagger}(x) \rightarrow \psi^{a \dagger}(x) e^{-i \Lambda(x)}
\end{array}
$$

are generated by the operator

$$
\mathcal{G}_{\Lambda} \equiv \sum_{x}\left(-\nabla_{i} \Lambda(x) E_{i}(x)+\Lambda(x)\left(\psi^{a \dagger}(x) \psi^{a}(x)-N_{\mathrm{L}} / 2\right)\right)
$$

The local generator of gauge transformations where $\Lambda$ has compact support is

$$
\frac{\partial \mathcal{G}_{\Lambda}}{\partial \Lambda(x)} \equiv \mathcal{G}(x)=\hat{\nabla} \cdot E(x)+\psi^{a \dagger}(x) \psi^{a}(x)-N_{\mathrm{L}} / 2
$$

Both (56) and (57) commute with the Hamiltonians in (53).

In (56) and (57) we have subtracted the constant $N_{\mathrm{L}} / 2$ from the charge density operator in order to make the gauge generator odd under the usual charge conjugation transformation

$$
\begin{array}{ll}
\xi: & A_{i}(x) \rightarrow-A_{i}(x) \\
\xi: & E_{i}(x) \rightarrow-E_{i}(x) \\
\xi: & \psi^{a}(x) \rightarrow(-1)^{\sum_{k=1}^{D} x_{k}} \psi^{a \dagger}(x) \\
\xi: & \psi^{a \dagger}(x) \rightarrow(-1)^{\sum_{k=1}^{D} x_{k}} \psi^{a}(x)
\end{array}
$$

In fact, the Fermionic charge term in (56) can be put in the manifestly odd form $\frac{1}{2}\left[\psi^{a \dagger}(x), \psi^{a}(x)\right]$. Of course, charge conjugation symmetry of the lattice theory is necessary to obtain charge conjugation of the continuum theory. We shall see later that, particularly at strong coupling, the subtraction term in (56) plays an important role. It seems to have been ignored in previous literature (for example, see [27, 25). Its presence is particularly important 
when $N_{\mathrm{L}}$ is odd since the charge operator has no zero eigenvalues in that case (the eigenvalues of $\psi^{a^{\dagger}}(x) \psi^{a}(x)$ are integers).

Chiral symmetry is related to translation invariance by one site. The Hamiltonian (53) is invariant under the transformations

$$
\begin{array}{ll}
\chi_{j}: & A_{i}(x) \rightarrow A_{i}(x+\hat{j}) \\
\chi_{j}: & E_{i}(x) \rightarrow E_{i}(x+\hat{j}) \\
\chi_{j}: & \psi^{a}(x) \rightarrow(-1)^{\sum_{k=j+1}^{D} x_{k}} \psi^{a}(x+\hat{j}) \\
\chi_{j}: & \psi^{a \dagger}(x) \rightarrow(-1)^{\sum_{k=j+1}^{D} x_{k}} \psi^{a \dagger}(x+\hat{j})
\end{array}
$$

for $j=1, \cdots, D$.

In the following we shall use the charge conjugation symmetry which is a combination of these two transformations:

$$
C \equiv \xi \chi_{1}
$$

This is necessary if the mass operators which we defined in the previous section are to be invariant under charge conjugation symmetry. Also, we shall see that the strong coupling ground state is invariant under $C$ but not under either $\xi$ or $\chi_{j}$ by themselves.

The dynamical problem of Hamiltonian lattice gauge theory is to find the eigenstates of the Hamiltonian operator (53) and out of those eigenstates to find the ones which are gauge invariant, i.e. which obey the physical state condition (or, the "Gauss' law" constraint)

$$
\mathcal{G}(x) \mid \Psi_{\text {phys. }}>=0
$$

In the case of compact QED, the periodicity of the gauge potential induces an additional symmetry which, being a large gauge symmetry, can be represented projectively. In the Schrödinger picture we shall require that the quantum states which are functions of a configuration of the gauge field transform as

$$
\left|A_{i}(x)+2 \pi n>=\exp (i n \theta[x, i])\right| A_{i}(x)>
$$

There is a separate parameter $\theta[x, i]$ for each link of the lattice. These parameters originate in a way similar to the theta-angle in ordinary QCD. The 
symmetry (62) together with the commutator (51) imply that the spectrum of the electric field operator has eigenvalues which are separated by integers and offset by $\theta$ :

$$
\operatorname{spectrum}\left[E_{i}(x)\right]=\{\theta[x, i]+\text { integers }\}
$$

The Hamiltonian and gauge constraints can be obtained from the gauge invariant Lagrangian

$$
\begin{array}{r}
L=\sum_{x} \psi^{a \dagger}(x)\left(i \partial_{t}-A_{0}(x)\right) \psi^{a}(x)+\sum_{[x, i]} E_{i}(x) \dot{A}_{i}(x) \\
+\sum_{[x, i]} E_{i}(x) \nabla_{i} A_{0}(x)+\sum_{x} A_{0}(x) N_{\mathrm{L}} / 2-H
\end{array}
$$

where the temporal component of the gauge field has been introduced to enforce the gauge constraint and the time derivative terms give the correct symplectic structure. In order to get Lorentz invariance of the Fermion spectrum in the weak coupling (naive) continuum limit, we require half-filling of the Fermionic states, i.e. that the total charge defined by

$$
\sum_{x}\left(\psi^{a \dagger}(x) \psi^{a}(x)-N_{\mathrm{L}} / 2\right)
$$

has zero vacuum expectation value.

Here we have considered massless QED. As well as the gauge invariance and charge conjugation invariance discussed above, the Hamiltonian is sym-

metric under the discrete chiral transformations (59) which on the lattice corresponds to symmetry under translation by one site. In later Sections, we shall consider the possibility of spontaneous breaking of this symmetry.

\subsection{Gauge fixing and quantization}

We shall quantize the gauge fields in the Schrödinger picture. The quantum states are functions of the link operators which are represented by the classical variables $A_{i}(x)$ and the electric field operators are derivatives

$$
E_{i}(x) \equiv-i \frac{\partial}{\partial A_{i}(x)}
$$


We shall also consider the usual Fock representation of the Fermion anticommutator. The empty vacuum is the cyclic vector

$$
\psi^{a}(x) \mid 0>=0 \quad \forall a, x
$$

and Fermions occupying lattice sites are created by $\psi^{a \dagger}(x)$.

In compact QED the spectrum of the gauge generator is discrete and a state which obeys the physical state condition can be normalized, thus implying that there is no need for additional gauge conditions. The basis wave-functions for compact QED (in the basis where the Fermions density and eletric field operators are diagonal) are $\Psi[n(x)] \Phi[A]$ with the Fermion states

$$
\Psi[n(x)]=\prod_{x} \prod_{a=1}^{N_{\mathrm{L}}}\left(\psi^{a \dagger}(x)\right)^{n_{a}(x)} \mid 0>
$$

labeled by vectors $n(x)=\left(n_{1}(x), \cdots, n_{N_{\mathrm{L}}}(x)\right)$ with $n_{a}(x)=0$ or 1 , and the photon states

$$
\Phi[A]=\exp \left(i \sum e_{i}(x) A_{i}(x)\right)
$$

where the eigenvalues $e_{i}(x)$ of the electric field operator are in spectrum $\left[E_{i}(x)\right]$ (63). Furthermore the states of the photon field are normalized using the inner product

$$
<\Phi_{1}[A], \Phi_{2}[A]>=\prod_{[x, i]} \int_{0}^{2 \pi} \frac{d A_{i}(x)}{2 \pi} \Phi_{1}^{\dagger}[A] \Phi_{2}[A]
$$

and the Fermion states have conventional inner product given by $<0 \mid 0>=1$ and the canonical anticommutator relations of the fermion field operators. The physical state condition (61) gives the additional restriction that

$$
\hat{\nabla}_{i} e_{i}(x)=-\rho(x)=-\sum_{x} \sum_{a}\left(n_{a}(x)-1 / 2\right)
$$

where $\rho(x)$ is the charge density (i.e. the eigenvalue of $\psi^{a \dagger}(x) \psi^{a}(x)-N_{\mathrm{L}} / 2$ ). Pictorially, we can think of this as containing lines of electric flux joining sites whose charges are non-zero and also closed loops of electric flux. In the strong coupling limit, the Hamiltonian is diagonal in the basis (68). This gives a 
natural explanation of confinement in compact QED in the strong coupling region. If we add a particle-antiparticle pair to a state in (68) it must be accompanied by at least a single line of electric flux. The energy of such a line of flux is proportional to its length. Therefore the electron-positron interaction grows linearly with distance and is confining. This is in contrast to the situation in non-compact QED where the electric flux is not quantized. In that case, a particle-antiparticle pair can have many lines with arbitrarily small flux. The energy of the field is minimized by the usual Coulomb dipole configuration. In high enough dimensions this is not a confining interaction.

\section{Strong Coupling Expansion}

In compact QED the eigenstates of the electric field operator are normalizable and can be used for the ground state. In this case we separate the Hamiltonian into three terms,

$$
\begin{array}{r}
H_{0}=\sum_{[x, i]} \frac{e^{2}}{2} E_{i}^{2}(x) \\
H_{1}=\sum_{[x, i]} t_{[x, i]}\left(\psi^{\dagger}(x+\hat{i}) e^{i A_{i}(x)} \psi(x)+\text { h.c. }\right) \\
H_{2}=\sum_{[x, i, j]} \frac{2}{e^{2}} \sin ^{2}(B[x, i, j] / 2)
\end{array}
$$

In the strong coupling limit it is necessary to solve Gauss' law (61) for the electric fields and the charge distribution in such a way as to minimize $H_{0}$.

When $N_{\mathrm{L}}$ is even, the charge operator has zero eigenvalues and Gauss' law has the solution where $E_{i}(x)=0$, which is an obvious minimum of the $H_{0}$, and there are $N_{\mathrm{L}} / 2$ fermions on each site. The degeneracy of this state may be resolved, resulting in an effective Hamiltonian which describes the $\mathrm{SU}\left(N_{\mathrm{L}}\right)$ antiferromagnet in the representation with Young Tableau having one column with $N_{\mathrm{L}} / 2$ boxes. This system has Néel order in $D \geq 2$ if $N_{\mathrm{L}}$ is small enough and the chiral symmetry of electrodynamics is broken, along with the $\mathrm{SU}\left(N_{\mathrm{L}}\right)$ flavor symmetry [2].

In order to see this we follow [2] and resolve the degeneracy by diagonalizing perturbations in the hopping parameter expansion. The first order 
perturbations vanish. The first non-trivial order is second order,

$$
\delta_{2}=-<g . s .\left|H_{1} \frac{1}{H_{0}-E_{0}} H_{1}\right| g . s .>
$$

This matrix element can be evaluated by noting that $H_{1}$ creates an eigenstate of $H_{0}$ different from the ground states with additional energy

$$
\Delta E=\frac{e^{2}}{2}+\frac{e^{2}}{2}(D) \nabla_{1}\left(x\left|\frac{1}{-\nabla \cdot \hat{\nabla}}\right| x\right)=e^{2}
$$

Diagonalizing the matrix of second order perturbations is equivalent to finding the spectrum of the effective Hamiltonian

$$
\begin{array}{r}
H_{\mathrm{eff}}=\frac{2|t|^{2}}{e^{2}} \sum_{[x, i]} \psi^{b \dagger}(x+\hat{i}) \psi^{b}(x) \psi^{a \dagger}(x) \psi^{a}(x+\hat{i}) \\
=\frac{2|t|^{2}}{e^{2}} \sum_{[x, i]} J_{a b}(x) J_{b a}(x+\hat{i})
\end{array}
$$

where the operators $J_{a b}(x)=\psi^{a \dagger}(x) \psi^{b}(x)-\frac{1}{2} \delta_{a b}$, are the generators of the $\mathrm{U}\left(N_{\mathrm{L}}\right)$ given in equation (42) and obeying the Lie algebra in equation (41).

The constraint on the total occupation number of each site,

$$
\rho(x)=\sum_{a=1}^{N_{L}} J_{a a}(x) \sim 0
$$

reduces to $\mathrm{SU}\left(N_{\mathrm{L}}\right)$ and projects onto a fundamental representations of $\mathrm{SU}\left(N_{\mathrm{L}}\right)$ [2].

It is straightforward to see that the higher orders in the hopping parameter expansion also have higher orders of $1 / e^{2}$. In fact, if we consider the following limit,

$$
\begin{array}{r}
e^{2} \rightarrow \infty, \quad|t|^{2} \rightarrow \infty \\
|t|^{2} / e^{2}=\text { constant }
\end{array}
$$

all higher order perturbative contributions to both the wavefunction and the energy vanish. Thus, in this limit, QED is exactly equivalent to an $\mathrm{SU}\left(N_{\mathrm{L}}\right)$ antiferromagnet. That (75) is the correct limit to take can be seen from the 
fact that, if the antiferromagnet in (74) is in an ordered state, the speed of the spin-waves, which are the gapless low-energy excitations is proportional to $|t| / e$. They have linear dispersion relation $\omega(k) \sim|k|$ and play the role of massless goldstone bosons for broken flavor symmetry. Their speed should be equal to the speed of light, which is one in our units. This implies that $|t| / e$ should be adjusted so that the spin-wave spectrum is relativistic, $\omega(k)=|k|$. Hence the limit in (75).

When $N_{\mathrm{L}}=2$, this model is the quantum Heisenberg antiferromagnet in the $j=1 / 2$ representation. It is known to have a Néel ordered ground state in $D \geq 3$ [28] and there is good numerical evidence that it has Néel order in $D=2$. The antiferromagnetic order parameter is the mass operator

$$
\vec{\Sigma}=\sum_{x}(-1)^{\sum_{i=1}^{D} x_{i}} \psi^{\dagger}(x) \vec{\sigma} \psi(x)
$$

which obtains a vacuum expectation value in the infinite volume limit. Thus, when $N_{L}=2$ the strong coupling limit breaks chiral symmetry and generates electron mass. It is interesting that in this case there is an iso-vector condensate. In the strong coupling limit this seems unavoidable. The only way to get an iso-scalar condensate is with a charge density wave. However such a configuration always has infinite coulomb energy compared to an electric charge neutral but isospin carrying condensate.

The low energy excitations of this systems (with energies of order $|t|^{2} / e^{2}$ are spin waves. All other excitations have energies which go to infinity in the limit (75). The spin waves are the pions which are the scalar Goldstone bosons arising from spontaneous breaking of the vector flavor symmetry $\mathrm{SU}(2) \rightarrow \mathrm{U}(1)$.

For large $N_{\mathrm{L}}$ there is some evidence that the $\mathrm{SU}\left(N_{\mathrm{L}}\right)$ antiferromagnet in these specific representations has a disordered ground state [29]. Particularly in 2+1-dimensions it is known that for infinite $N_{L}$ the ground state is disordered [5].

When $N_{\mathrm{L}}$ is odd, since the charge density operator has no non-zero eigenvalues, it is impossible to find a zero eigenstate of the Gauss' law constraint operator without some electric field. The problem which we must solve is to minimize the energy functional $\sum E^{2}$ subject to the constraint $\hat{\nabla} \cdot E=-\rho$ where at each site $\rho$ has one of the values

$$
-\frac{N_{\mathrm{L}}}{2},-\frac{N_{\mathrm{L}}}{2}+1, \ldots, \frac{N_{\mathrm{L}}}{2} \quad N_{\mathrm{L}} \text { an odd integer }
$$


The reader can easily convince herself that the charge distribution and electric field which one obtains are [2],

$$
\rho_{0}(x)=\frac{1}{2}(-1)^{\sum_{i=1}^{D} x_{i}} \quad E_{i}(x)=\frac{1}{4 D}(-1)^{\sum_{i=1}^{D} x_{i}}
$$

These configurations break chiral symmetry in that they are not invariant under the transformation $\chi_{1}$ in (59) but they are symmetric under charge conjugation $C$ defined in (60).

The ground state energy per lattice site is

$$
\frac{E_{0(\mathrm{coul})}}{|V|}=\frac{e^{2}}{32 D}
$$

Note that it is of order $e^{2}$. This is in contrast to the ground state energy when the number of lattice Fermion flavors is even, which is of order $|t|^{2} / e^{2} \sim 1$.

The ground states that we have found are highly degenerate in that only the number of Fermions at each site is fixed. Their quantum state can still take up any orientation in the vector space which carries the representation of the flavor $\mathrm{SU}\left(N_{\mathrm{L}}\right)$ given by the Young Tableaux with one column and $\left(N_{\mathrm{L}}-1\right) / 2$ or $\left(N_{\mathrm{L}}+1\right) / 2$ rows on each of the two sublattices $A$ and $B$ in which we have divided the lattice. Namely : $A$ is the set of all points where $\sum_{i} x_{i}$ is even and $B$ the one where $\sum_{i} x_{i}$ is odd. The differing occupation numbers on sites on each sublattice yield different representations of $\mathrm{SU}\left(N_{L}\right)$.

The degeneracy must be resolved by diagonalizing the perturbations, which are non-zero in second order and the problem is equivalent to diagonalizing the antiferromagnet Hamiltonian (74). Also, in the limit (75) this correspondence is exact.

The strong coupling ground states that we find when $N_{\mathrm{L}}$ is odd contains a charge density wave. The staggered charge density operator has expectation values

$$
\frac{1}{|V|}<\sum_{x}(-1)^{\sum_{k=1}^{D} x_{k}} \psi^{\dagger}(x) \psi(x)>= \pm \frac{1}{2}
$$

This condensate is an isoscalar and we have shown that it must always occur in all dimensions. When $N_{\mathrm{L}}>1$ mass operators with certain generators of $\mathrm{SU}\left(N_{\mathrm{L}}\right)$ could have expectation values if the ground state has antiferromagnetic order. However, unlike the case of even $N_{\mathrm{L}}$, the antiferromagnetic order is not required in order to have chiral symmetry breaking. 
The ground state we find breaks chiral symmetry. This is a true dynamical symmetry breaking since, in infinite volume, the ground states which are related by a chiral transformation are never mixed in any order of strong coupling perturbation theory. Furthermore, there are no local operators which couple them.

We conclude that the strong coupling ground state breaks chiral symmetry for any odd $N_{\mathrm{L}}$ and in any dimensions. As in the case of even $N_{L}$ there is also the possibility (and for small $N_{L}$ the likelyhood) that the $\mathrm{SU}\left(N_{L}\right)$ antiferromagnet we obtain here is in a Néel state and the flavor symmetry is also broken [29].

Notice that in the ground state, the electric fields are not integers, but on each link, the spectrum of the electric field operator is $1 / 4 D+$ integers. The "theta angles" 1/4D survive all orders in strong coupling perturbation theory.

\section{Remarks}

In this paper we reviewed our approach [2] to analyze the strong coupling limit of quantum electrodynamics using the Hamiltonian picture and a lattice regularization. We showed also the mechanism for chiral symmetry breaking in this lattice gauge theory. We chose to use staggered Fermions because they give the closest analog to interesting condensed matter physics systems.

In $1+1$ dimensions, staggered Fermions give $N_{\mathrm{L}}$ species of 2- component Dirac Fermions. When $N_{\mathrm{L}}=1$ we obtain the Schwinger model with a lattice regularization. Also, in this case, we have found that the chiral symmetry is broken dynamically. Of course, due to the staggered Fermion regularization there is no continuous chiral symmetry, which is as it should be since it should be impossible to regularize the Schwinger model so that there is simultaneous continuous chiral and gauge symmetry. However, to match the solution of the continuum Schwinger model, the Fermion should obtain mass. This indeed happens in our strong coupling limit by spontaneous symmetry breaking. (Although we disagree with some aspects of the formalism, we agree with the results of reference [27] on this point.)

This result should not be confined to strong coupling, but should persist for all coupling, i.e. the critical coupling in $D=1$ should be at $e^{2}=0$. We conjecture that this sort of symmetry breaking for small $e^{2}$ is a manifestation 
of the Peierls instability — the tendency of a one dimensional Fermi gas to form a gap at the Fermi surface. This happens with any infinitesimal interaction.

In fact, this must also happen for the case where $N_{\mathrm{L}}$ is even. Then, there cannot be any spin order in 1 dimension. However, anomalies break the isoscalar chiral symmetry in the continuum theory and should also do so here. This means that there should be a dynamical generation of charge density wave which would be driven by the Peierls insability. It also implies that for $N_{\mathrm{L}}=2$ for example, the ground state in the strong coupling limit would not be a Heisenberg antiferromagnet, but would be alternating empty site and site with two electrons in a spin singlet state. This state, even though it has large coulomb energy, avoids the infrared divergences of gapless Fermions.

In higher dimensions, $D \geq 2$, one should explore the possibility of phase transitions between different symmetry breaking patterns for the $\mathrm{SU}\left(N_{\mathrm{L}}\right)$ flavor symmetry as one varies $N_{\mathrm{L}}$. There is already some work on this subject in the condensed matter physics literature on $\mathrm{SU}\left(N_{\mathrm{L}}\right)$ antiferromagnets [29]. They analyze the $\mathrm{SU}\left(N_{\mathrm{L}}\right)$ antiferromagnet which is similar to the strong coupling limit of an $\mathrm{U}\left(N_{\mathrm{C}}\right)$ gauge theory (see 2] for details) which is in the representation corresponding to a rectangular Young Tableau with $N_{\mathrm{L}}$ rows and $N_{\mathrm{C}}$ columns. They work in the large $N_{\mathrm{C}}$ limit and show that there is a phase transition from the spin ordered Néel phase to a disordered phase when $N_{\mathrm{L}} \sim N_{\mathrm{C}}$. For QED $N_{\mathrm{C}}=1$ so their analysis is not accurate. For odd $N_{\mathrm{L}}$ the chiral symmetry is always broken and the question we are asking is whether the flavor symmetry is also broken. For even $N_{\mathrm{L}}$ possible phase transition is relevant to both chiral and flavor symmetry breaking. In this context it is interesting to ascertain if the order-disorder phase transition which occurs as one increases $N_{\mathrm{L}}$ in the $\mathrm{SU}\left(N_{\mathrm{L}}\right)$ antiferromagnet is the same one that appears in the study of chiral symmetry breaking in 2+1-dimensional QED in the continuum [19, 20] where they find that chiral symmetry is broken only if the number of flavors is less than a certain critical value. These questions have been addressed in 18.

Our study suggests that, besides the critical $N_{\mathrm{L}}$, for a fixed $N_{\mathrm{L}}$ which is small enough, there should also be a critical coupling constant $e^{2}$ and, in fact, a critical line in the $N_{\mathrm{L}}-e^{2}$ plane where there is a second order phase transition between a spin ordered chiral symmetry breaking phase and a disordered (and possibly chirally symmetric phase). We speculate that in $3+1$-dimensions a similar situation could occur. The latter phenomenon 
has recently been discussed in the context of $2+1$-dimensional gauge theory [18]. It can in principle shed light on the nature of the phase transition in the lattice theories. It would be interesting to analyze the critical behavior further, particularly in the lattice theory, since one could address the question of whether this phase transition is the same as the one seen in Josephson junction arrays.

We have seen that the Coulomb gas representation of classical Josephson junction arrays is described at infinite coupling by the $N_{L}=1$ Coulomb gas. Away from infinite coupling in the case of electrodynamics and away from the classical limit in the case of junctions arrays the two systems may still have many common features.

In the quantum regime, Josephson junction arrays may be modeled by the quantum frustrated XY model which is equivalent to a model of lattice electrons in a magnetic field determined by the charge density [12]. In a mean field theory, where the charge density is replaced by its average, this resembles the Azbel-Wannier-Hofstaeder problem for lattice electrons with nearest neighbor hopping with an external magnetic field [12. When $f=$ $1 / 2$ the low energy components of the electron spectrum are approximately described by a massless Dirac Hamiltonian. Thus, for $f=1 / 2$, the model has relativistic lattice electrons and is gauge and parity invariant. When $f$ is a rational number, the frustrated quantum XY model admits a flux phase as its mean field theory ground state [12]. It would be interesting to analyze the properties of this model beyond mean field theory. In particular to ascertain if - for $f$ rational - the flux phase may be regarded as the exact ground state [30] as well as to investigate if, for $f=1 / 2$, quantum fluctuations could reproduce the relevant operators of compact QED. 


\section{References}

[1] G. W. Semenoff, Mod. Phys. Lett. A17, (1992) 2811; E. Langmann and G. W. Semenoff, Phys. Lett. B297 (1992), 175.

[2] M.C. Diamantini, E. Langmann, G.W. Semenoff, P. Sodano," SU(N) Antiferromagnets and the Phase Structure of QED in the Strong Coupling Limit" , DFUPG 69-92,December 1992;

M. C. Diamantini, P. Sodano, E. Langmann and G. W. Semenoff, "Quantum Spin Systems and the Vacuum Structure of Strong Coupling Gauge Theory with Dynamical Fermions", Proceedings of Field Theory and Collective Phenomena, Perugia, Italy, June 1992.

[3] J. Smit, Nucl. Phys. B175, (1980) 307.

[4] E. Fradkin, Field Theories and Condensed Matter Systems, Frontiers in Physics, Addison-Wesley Publishing Company, 1991.

[5] I. Affleck and B. Marston, Phys. Rev. B37, (1988) 3774.

[6] I. Affleck, Z. Zou, T. Hsu, and P.W. Anderson,Phys. Rev.B38, (1988) 745 .

[7] D. Hofstaeder, Phys.Rev. B14, (1976) 2239.

[8] R. Rammel, T.C. Lubensky and G. Toulouse, Phys.Rev. B27, (1983) 2820.

[9] W. Shih and D. Stroud, Phys. Rev. B28, (1983) 6575.

[10] T. C. Halsey, Phys. Rev. B31, (1985) 5728;

T. C. Halsey, J. Phys. C 18, (1985) 2437.

[11] D. Schmeltzer, A.R. Bishop, Phys. Rev.B41, (1990) 9603.

[12] M.C. Diamantini, P. Sodano, Phys.Rev.B45, (1992) 5737.

[13] J. Villain, J. Phys. 36 (1975), 581; J.V. Jose, L. P. Kadanoff, S. Kirkpatrick and D. R. Nelson, Phys. Rev. B16 (1977), 121.

[14] A. R. Bishop and P. S. Lomdahl, Phys. Rev. B41 (1990), 10983. 
[15] C. Bruder, R. Fazio, A. Kampf, A. van Otterlo and G. Schön, 'Quantum phase transitions and commensurability in frustrated Josephson junction arrays", Karlsrue preprint, 1992.

[16] P. Fomin, V. Gusynin, V. Miranski and Yu. Sitenko, Riv. Nuov. Cim. $6,(1983) 1$.

[17] V. Miransky, Nuov. Cim. 90A, (1985) 149.

[18] M.C. Diamantini, G.W. Semenoff, P. Sodano ," Chiral Symmetry Breaking in Three Dimensional Gauge Theories" , DFUPG 70-92,December 1992

[19] Applequist, D. Nash and L. C. R. Wijewardhana, Phys. Rev. Lett. 60, (1988) 2575.

[20] D. Nash, Phys. Rev. Lett. 62, (1989) 3024.

[21] J. Kogut, Proceeding of Lattice'92, Amsterdam, June, 1992; E. Dagotto, J. Kogut and A. Kocic, Phys. Rev. Lett. 62, (1992) 1083.

[22] A. Polyakov, Nucl. Phys. B120, (1977) 429.

[23] A. J. Niemi and G. W. Semenoff, Phys. Rev. Lett. 51, (1983) 2077; A. N. Redlich, Phys. Rev. Lett. 52, (1984) 1.

[24] H. Kluberg-Stern, A. Morel, O. Napoly and B. Petersson, Nucl. Phys. B220 [FS8], (1983) 447.

[25] J. B. Kogut, Rev. Mod. Phys. 55, (1983) 775.

[26] J. B. Kogut and E. Dagotto, Phys. Rev. Lett. 59, (1987) 617.

[27] A. Caroll, J. Kogut, D. K. Sinclair and L. Susskind, Phys. Rev. D8, (1976) 2270;

T. Banks, L. Susskind and J. Kogut, Phys. Rev. D4, (1976) 1043.

[28] T. Kennedy, E. H. Lieb and B. S. Shastry, J. Stat. Phys. 53, (1988) 1019. 
[29] N. Read and S. Sachdev, Phys. Rev. Lett. 62 (1989) 1694;

N. Read and S. Sachdev, Phys. Rev. B42 (1990) 4568;

N. Read and S. Sachdev, Nucl. Phys. B316 (1990) 609.

[30] I. Affleck, D.P. Arovas, J.B. Marston, D.A. Rabson Nucl.Phys. B366 (1991) 467. 